TRANSACTIONS OF THE

AMERICAN MATHEMATICAL SOCIETY

Volume 364, Number 5, May 2012, Pages 2461-2478

S 0002-9947(2012)05372-4

Article electronically published on January 13, 2012

\title{
$D$-MODULE STRUCTURE OF LOCAL COHOMOLOGY MODULES OF TORIC ALGEBRAS
}

\author{
JEN-CHIEH HSIAO
}

\begin{abstract}
Let $S$ be a toric algebra over a field $\mathbb{K}$ of characteristic 0 and let $I$ be a monomial ideal of $S$. We show that the local cohomology modules $H_{I}^{i}(S)$ are of finite length over the ring of differential operators $D(S ; \mathbb{K})$, generalizing the classical case of a polynomial algebra $S$. As an application, we compute the characteristic cycles of some local cohomology modules.
\end{abstract}

\section{INTRODUCTION}

Lyubeznik Lyu93 introduced an approach of studying local cohomology modules using the theory of $D$-modules. He obtained many finiteness properties of the local cohomology modules $H_{I}^{i}(R)$ when $R$ is a regular ring containing a field of characteristic 0. For example, taking advantage of holonomicity of $H_{I}^{i}(R)$ as a $D$-module, he showed that for any maximal ideal $\mathfrak{m}$ the number of associated prime ideals of $H_{I}^{i}(R)$ contained in $\mathfrak{m}$ is finite and that all Bass numbers of $H_{I}^{i}(R)$ are finite. When $R$ is a regular local ring of positive characteristic, analogous results were obtained by Huneke and Sharp using the Frobenius functor HS93. When $R$ is not regular, the situation is more subtle. There are characteristic-free examples where $H_{I}^{i}(R)$ have infinitely many associated primes SS04. Also, an example by Hartshorne [Har70] shows that in general the Bass numbers can be infinite (see Example 3.9).

After Lyu93, there have been several studies on the finiteness properties of $R_{x}$ and of $H_{I}^{i}(R)$ as $D$-modules for a regular ring $R$, among them Bøg95, Bøg02, Lyu97, ÁMBL05. The first $D$-finiteness result of $R_{x}$ for a singular ring $R$ is due to Takagi and Takahashi [TT08] which says $R_{x}$ is generated by $x^{-1}$ over $D$ when $R$ is a Noetherian graded ring with finite F-representation type. In particular, their theorem applies to the case where $R$ is a normal toric algebra over a perfect field of positive characteristic.

In the present article, we study finiteness properties of the localizations $S_{f}$ and the local cohomology modules $H_{I}^{i}(S)$ as $D$-modules, where $S$ is a toric algebra (not necessarily normal) over a field $\mathbb{K}$ of characteristic $0, f$ is a monomial element and $I$ is a monomial ideal of $S$. In this case, the ring of differential operators $D(S):=D(S ; \mathbb{K})$ is much more complicated than the case where $S$ is regular. Using the natural grading of $D(S)$ introduced by Jones Jon94 and Musson Mus94, Saito and Traves [ST01, ST04 gave a detailed description of $D(S)$. Based on their results, we prove that any localization $S_{f}=S\left[f^{-1}\right]$ of $S$ is generated by $f^{-1}$ over $D(S)$

Received by the editors December 17, 2009 and, in revised form, April 8, 2010 and April 14, 2010.

2010 Mathematics Subject Classification. Primary 13D45, 13N10, 14M25.

The author was partially supported by the NSF under grants DMS 0555319 and DMS 0901123.

(C)2012 American Mathematical Society

Reverts to public domain 28 years from publication 
(Theorem 3.1). This implies immediately that $H_{I}^{i}(S)$ is $D(S)$-finitely generated if $D(S)$ is left Noetherian. Unfortunately, $D(S)$ is not always left Noetherian [ST09]. Nonetheless, we can show that $H_{I}^{i}(S)$ is actually of finite length as a $D(S)$-module (Theorem 3.3). In view of Hartshorne's example (Example 3.9), this result is quite surprising.

As an application, we compute the characteristic cycles of some local cohomology modules $H_{I}^{i}(S)$. Characteristic cycles are formal sums of subvarieties (counted with multiplicities) of the characteristic variety of a $D$-module $M$. Here, the characteristic variety $\mathrm{Ch}(M)$ is the support of the associated graded module gr $M$ in the spectrum $\operatorname{Spec}(\operatorname{gr} D(S))$ of the associated graded ring gr $D(S)$. When $S$ is a polynomial algebra, one can explicitly compute the Bass numbers and the associated primes of $H_{I}^{i}(S)$ from its characteristic cycles ÁM04. The cohomological dimension of $I$ and the Lyubeznik numbers can also be computed from them ÁM00. Through our finiteness results of $H_{I}^{i}(S)$, we are able to compute the characteristic cycles of some local cohomology modules. We will show that for normal toric algebras $S$ (in fact, for a more general class of toric algebras) the characteristic variety $\mathrm{Ch}\left(H_{\mathfrak{m}}^{\operatorname{dim} S}(S)\right)$ of the top local cohomology with maximal support is abstractly isomorphic to the ambient toric variety $\operatorname{Spec}(S)$ (Theorem 4.13).

In section 2, we briefly recall the notions of local cohomology, toric algebras and the ring of differential operators of a commutative algebra over a field. We also describe the structure of rings of differential operators over toric algebras following the notation in ST01 and ST04. In section 3, our main results on the finiteness properties mentioned above are presented. Also, we relate our finiteness results to the notion of sector partition introduced in [SS90] and MM06. Some discussions on gr $D(S)$ and the computations of characteristic cycles are in section 4 . As suggested by the referee, some relations between our results in section 4 and the recent work of Saito [Sai10, are discussed (see Remarks 4.7, 4.14).

\section{Preliminaries}

2.1. Local cohomology. General facts regarding local cohomology can be found in ILL+07 or [BS98. Here, we only recall some basics.

Let $R$ be a Noetherian commutative ring, $M$ an $R$-module, and $I$ an ideal of $R$. Define $\Gamma_{I}(M):=\lim _{\operatorname{Hom}}\left(R / I^{k}, M\right)$. Then $\Gamma_{I}$ is a left exact $R$-linear covariant functor and the $i$-th local cohomology functor $H_{I}^{i}$ is defined to be its $i$-th right derived functor. We call $H_{I}^{i}(M)$ the $i$-th local cohomology module of $M$ supported at the ideal $I$. If $I$ is generated by $f_{1}, \ldots, f_{t}$, then $H_{I}^{i}(M)$ is the $i$-th cohomology of the Čech complex

$$
0 \rightarrow M \rightarrow \bigoplus_{i=1}^{t} M_{f_{i}} \rightarrow \bigoplus_{1 \leq i<j \leq t} M_{f_{i} f_{j}} \rightarrow \cdots \rightarrow M_{f_{1} \cdots f_{t}} \rightarrow 0 .
$$

2.2. Toric algebras. We introduce some notation for later use. For more information on toric algebras, the reader is referred to Ful93, [MS05] or [ILL+07].

Let $A$ be a $d \times n$ integer matrix with columns $a_{1}, \ldots, a_{n}$. Assume $\mathbb{Z} A=\mathbb{Z}^{d}$. For a field $\mathbb{K}$, the semigroup subring $S_{A, \mathbb{K}}:=\mathbb{K}[\mathbb{N} A]=\mathbb{K}\left[t^{a_{1}}, \ldots, t^{a_{n}}\right]$ of the Laurent polynomial ring $\mathbb{K}\left[\mathbb{Z}^{d}\right]=\mathbb{K}\left[t_{1}^{ \pm 1}, \ldots, t_{d}^{ \pm 1}\right]$ is called the toric algebra associated to the matrix $A$. Denote $\widetilde{\mathbb{N} A}:=\mathbb{R}_{\geq 0} A \cap \mathbb{Z}^{d}$ as the saturation of $\mathbb{N} A$. Then $\widetilde{S_{A, \mathbb{K}}}:=\mathbb{K}[\widetilde{\mathbb{N} A}]$ is the normalization of $S_{A, \mathbb{K}}$. 
2.3. Rings of differential operators. For a commutative algebra $R$ over a field $\mathbb{K}$, set $D_{0}(R ; \mathbb{K}):=R$, and for $i>0$,

$$
D_{i}(R ; \mathbb{K}):=\left\{f \in \operatorname{Hom}_{\mathbb{K}}(R, R) \mid[f, r] \in D_{i-1}(R ; \mathbb{K}) \text { for all } r \in R\right\} .
$$

Then the ring of differential operators is defined to be

$$
D(R ; \mathbb{K}):=\bigcup_{j} D_{j}(R ; \mathbb{K}) .
$$

When $R$ is a polynomial ring over a field $\mathbb{K}$ of characteristic $0, D(R ; \mathbb{K})$ is the usual Weyl algebra. In this paper, a module over $D(R ; \mathbb{K})$ means a left $D(R ; \mathbb{K})$-module.

Lemma 2.1. If $M$ is a $D(R ; \mathbb{K})$-module and $f \in R$, then the $R$-module structure on $M_{f}$ extends uniquely to a $D(R ; \mathbb{K})$-module structure such that the natural map $M \rightarrow M_{f}$ is a $D(R ; \mathbb{K})$-module homomorphism. In particular, via the Čech complex $H_{I}^{i}(M)$ has a natural $D(R ; \mathbb{K})$-module structure.

Proof. See Lyu00, Example (b).

When $R$ is a regular algebra over a field $\mathbb{K}$ of characteristic $0, D(R ; \mathbb{K})$ is well understood (see e.g. Bjö79]). In this case, the local cohomology modules $H_{I}^{i}(R)$ are holonomic as $D(R ; \mathbb{K})$-modules and hence are of finite length (see Lyu93). This essential property enables Lyubeznik to achieve many finiteness results of the local cohomology modules.

Unfortunately, $D(R ; \mathbb{K})$ does not behave well when $R$ is singular; we don't have a notion of holonomicity in this case. This complicates the study of $H_{I}^{i}(R)$ via the theory of $D$-modules. On the bright side, when $R=S_{A, \mathbb{K}}$ is a toric algebra over an algebraically closed field $\mathbb{K}$ of characteristic 0 , there is a nice combinatorial structure for $D(R ; \mathbb{K})$ which we will present in the next subsection. Our finiteness results about local cohomology modules substantially rely on this structure.

2.4. Rings of differential operators over toric algebras. In the rest of this paper, we denote $S_{A}:=S_{A, \mathbb{K}}$, where $\mathbb{K}$ is an algebraically closed field of characteristic 0. Following [ST01, the noncommutative ring $D_{A}:=D\left(S_{A}, \mathbb{K}\right)$ can be described as a $\mathbb{Z}^{d}$-graded subring of

$$
D\left(\mathbb{K}\left[\mathbb{Z}^{d}\right] ; \mathbb{K}\right)=\mathbb{K}\left[t_{1}^{ \pm 1}, \ldots, t_{d}^{ \pm 1}\right]\left\langle\partial_{1}, \ldots, \partial_{d}\right\rangle,
$$

where $\left[\partial_{i}, t_{j}\right]=\delta_{i j},\left[\partial_{i}, t_{j}^{-1}\right]=-\delta_{i j} t_{j}^{-2}$ and the other pairs of variables commute. More precisely, with the notation $\theta_{i}:=t_{i} \partial_{i}$, one has

$$
D_{A}=\bigoplus_{a \in \mathbb{Z}^{d}} t^{a} \mathbb{I}(\Omega(a))
$$

where $\Omega(a)=\mathbb{N} A \backslash(-a+\mathbb{N} A)$ and $\mathbb{I}(\Omega(a))$ is the vanishing ideal of $\Omega(a)$ in $\mathbb{K}\left[\theta_{1}, \ldots, \theta_{d}\right]$.

\section{Finiteness properties of $H_{I}^{i}\left(S_{A}\right)$}

In this section, $\mathscr{F}$ will be denoted to be the set of all facets of $\mathbb{R}_{\geq 0} A$. For a face $\tau$ of $\mathbb{R}_{\geq 0} A$, we write $\mathbb{N}(A \cap \tau):=\mathbb{N} A \cap \mathbb{R} \tau$ and denote $\mathbb{Z}(A \cap \tau)$ as the group generated by $\mathbb{N}(A \cap \tau)$.

We recall some notation in [ST01, which are crucial to the proofs of Theorems 3.1 and 3.3 . 
For $a \in \mathbb{Z}^{d}$ and $\tau$ a face of $\mathbb{R}_{\geq 0} A$, define

$$
E_{\tau}(a):=\{l \in \mathbb{C}(A \cap \tau) \mid a-l \in \mathbb{N} A+\mathbb{Z}(A \cap \tau)\} / \mathbb{Z}(A \cap \tau) .
$$

Notice that

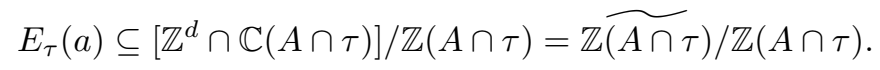

Here $\widetilde{\mathbb{Z}(A \cap \tau)}:=\mathbb{C}(A \cap \tau) \cap \mathbb{Z}^{d}$ is the saturation of $\mathbb{Z}(A \cap \tau)$, so each $E_{\tau}(a)$ is a finite set. Consider the ordering $\leq$ on $\mathbb{Z}^{d}$ defined by

$$
[a \leq b] \Longleftrightarrow\left[E_{\tau}(a) \subseteq E_{\tau}(b)\right] \text { for all faces } \tau \text { of } \mathbb{R}_{\geq 0} A .
$$

This ordering induces an equivalence relation on $\mathbb{Z}^{d}$ by

$$
[a \sim b] \Longleftrightarrow[a \leq b \text { and } b \leq a] .
$$

Now we are ready for the first main theorem.

Theorem 3.1. $S_{A}\left[f^{-1}\right]=D_{A} \cdot f^{-1}$ is cyclic as a left $D_{A}$-module for any monomial $f \in S_{A}$.

Proof. It's clear that $S_{A}\left[f^{-1}\right] \supseteq D_{A} \cdot f^{-1}$. Conversely, write $f=t^{b}$ for some $b \in \mathbb{N} A$ (we may assume $b \neq 0$ ). Since $S_{A}\left[f^{-1}\right] \subseteq D_{A} \cdot\left\{t^{-m b} \mid m \in \mathbb{N}\right\}$, it suffices to show that $t^{-m b} \in D_{A} \cdot t^{-b}$ for all $m \in \mathbb{N}$. By Proposition 4.1.5(1) in [ST01], it is enough to prove that $-b \sim-m b$. Indeed, we show that, for any face $\sigma, E_{\sigma}(-b)=E_{\sigma}(-m b)$ as follows:

(1) Let $\sigma$ be a facet, and suppose $b \notin \sigma$. Then $F_{\sigma}(-b)<0$, and hence $F_{\sigma}(-m b)<$ 0 . So $E_{\sigma}(-b)=E_{\sigma}(-m b)=\emptyset$.

(2) Let $\tau$ be a face and suppose that $b \notin \tau$. Then there exists a facet $\sigma$ containing $\tau$ with $b \notin \sigma$. Hence $E_{\sigma}(-b)=E_{\sigma}(-m b)=\emptyset$ by (1), and thus $E_{\tau}(-b)=E_{\tau}(-m b)=\emptyset$.

(3) Let $\tau$ be a face and suppose $b \in \tau$. Then $b \in \mathbb{N} A \cap \tau=\mathbb{N}(A \cap \tau)$. Hence, $\pm b \in \mathbb{Z}(A \cap \tau)$, and thus $E_{\tau}(-b)=E_{\tau}(-m b)$ by definition.

Remark 3.2. Via the Cech complex, it follows immediately from Theorem 3.1 that $H_{I}^{i}\left(S_{A}\right)$ is finitely generated over $D_{A}$ if $D_{A}$ is left Noetherian. The left Noetherianess of $D_{A}$ was studied by Saito and Takahashi [ST09]. They proved that $D_{A}$ is left Noetherian if $S_{A}$ satisfies Serre's condition $\left(S_{2}\right)$. Serre's condition is, by [Ish88], equivalent to

$$
S_{A}=\bigcap_{\tau: \text { facets }} \mathbb{K}[\mathbb{N} A+\mathbb{Z}(A \cap \tau)]
$$

Saito and Takahashi also gave a necessary condition (on $S_{A}$ ) for $D_{A}$ to be left Noetherian. However, $D_{A}$ is not always left Noetherian.

Nonetheless, we have

Theorem 3.3. For any $i$ and any monomial ideal $I, H_{I}^{i}\left(S_{A}\right)$ is of finite length as a $D_{A}$-module.

Proof. In view of the Čech complex, since any localization $S_{A}\left[f^{-1}\right]$ (with monomial

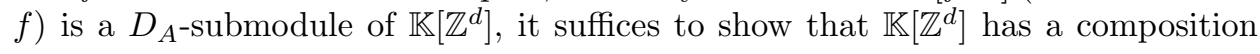
series. 
Consider the notation in the beginning of this section. For $a, b \in \mathbb{Z}^{d}$, we will write $a<b$ if $a \leq b$ but $a \nsim b$. Then, for each $a \in \mathbb{Z}^{d}, \bigoplus_{b \geq a} \mathbb{K} t^{b}$ is generated by $t^{a}$ as a $D_{A}$-module. Moreover,

$$
\frac{\bigoplus_{b \geq a} \mathbb{K} t^{b}}{\bigoplus_{b>a} \mathbb{K} t^{b}} \cong \bigoplus_{b \in[a]} \mathbb{K} t^{b}
$$

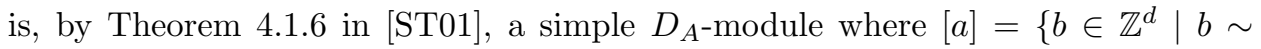
$a\}$. Since there are only finitely many faces and since each $E_{\tau}(a)$ is contained in

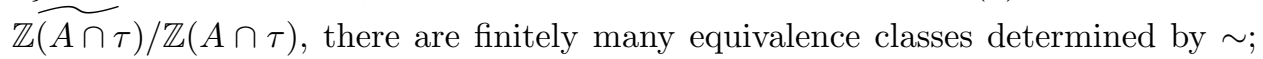
we denote them $\left[\alpha_{1}\right],\left[\alpha_{2}\right], \ldots,\left[\alpha_{k}\right]$. We may rearrange the order so that, for any pair $i<j$, either $\alpha_{i}>\alpha_{j}$ or $\alpha_{i}$ and $\alpha_{j}$ are incomparable. Denote $T_{a}:=\bigoplus_{b \geq a} \mathbb{K} t^{b}$. Then the filtration

$$
0 \subsetneq T_{\alpha_{1}} \subsetneq \cdots \subsetneq \Sigma_{l=1}^{i} T_{\alpha_{l}} \subsetneq \cdots \subsetneq \Sigma_{l=1}^{k} T_{\alpha_{l}}=\mathbb{K}\left[\mathbb{Z}^{d}\right]
$$

is a composition series of $D_{A^{-}}$submodules of $\mathbb{K}\left[\mathbb{Z}^{d}\right]$.

Example 3.4. For 1-dimensional $S_{A}$, the composition series of $\mathbb{K}[\mathbb{Z}]$ is easy to describe. In this case, $\mathbb{R}_{\geq 0} A$ has two faces, 0 and $\sigma=\mathbb{R}_{\geq 0} A$. For $a \in \mathbb{Z}$,

$$
\begin{gathered}
E_{0}(a)=\{\ell \in\{0\} \mid a-\ell \in \mathbb{N} A\} /\{0\} \text { and } \\
E_{\sigma}(a)=\{\ell \in \mathbb{Z} \mid a-\ell \in \mathbb{Z}\} / \mathbb{Z} .
\end{gathered}
$$

Thus $E_{0}(a)=\{0\}$ if $a \in \mathbb{N} A, E_{0}(a)=\emptyset$ if $a \notin \mathbb{N} A$, and $E_{\sigma}(a)=\{0\}$ for all $a \in \mathbb{Z}$. Therefore, $[0]$ and $[-1]$ are the two equivalence classes determined by $\sim$, and we have the composition series

$$
0 \subsetneq \mathbb{K}[\mathbb{N} A]=T_{0} \subsetneq T_{-1}=\mathbb{K}[\mathbb{Z}]
$$

Remark 3.5. Theorem 3.1 and Theorem 3.3 also hold for any field $\mathbb{K}$ with characteristic 0 by the isomorphism

$$
H_{I}^{i}\left(S_{A, \mathbb{K}}\right) \otimes \overline{\mathbb{K}} \cong H_{I}^{i}\left(S_{A, \overline{\mathbb{K}}}\right)
$$

Remark 3.6. Suppose $I=\mathfrak{m}$, the maximal graded ideal of $S_{A}$. Here we assume that the semigroup $\mathbb{N} A$ is pointed, so that 0 is the only invertible element.

(1) Recall that $H_{\mathfrak{m}}^{i}\left(S_{A}\right)$ can be computed as the $i$-th cohomology of the Ishida complex [Ish88] or ILL+07]. Therefore, $H_{\mathfrak{m}}^{1}\left(S_{A}\right)$ is finitely generated as an $S_{A}$-module. Indeed, it suffices to observe that

$$
H_{\mathfrak{m}}^{1}\left(S_{A}\right) \leftarrow \bigcap_{\sigma: \text { rays }} \mathbb{K}[\mathbb{N} A+\mathbb{Z}(A \cap \sigma)] \subseteq \bigcap_{\tau: \text { facets }} \mathbb{K}[\mathbb{N} A+\mathbb{Z}(A \cap \tau)] \subseteq \widetilde{S_{A}}
$$

and the fact that $\widetilde{S_{A}}$ is finite over $S_{A}$. Moreover, $H_{\mathfrak{m}}^{d}\left(S_{A}\right)$ is cyclic as a left $D_{A}$-module. This is because the $d$-th module in the Ishida complex is $\mathbb{K}\left[\mathbb{Z}^{d}\right]$, which is cyclic by Theorem 3.1 .

(2) In general, Schäfer and Schenzel SS90] showed that there is a partition of $\mathbb{Z}^{d}$ with respect to which $H_{\mathfrak{m}}^{i}\left(S_{A}\right)$ can be written as a finite direct sum of $\mathbb{K}$-vector spaces. This decomposition coincides with the sector partition appearing in [MM06] (see also [HM05] for a more general notion of sector partition). More precisely, let $\operatorname{Conv}(A)$ be the set of all faces of $\mathbb{R}_{\geq 0} A$, and for any filter (cocomplex) $\nabla$ of $\operatorname{Conv}(A)$, denote

$$
P_{\nabla}=\bigcap_{A \cap \tau \in \nabla}[\mathbb{N} A+\mathbb{Z}(A \cap \tau)] \backslash \bigcup_{A \cap \tau \notin \nabla}[\mathbb{N} A+\mathbb{Z}(A \cap \tau)]
$$


Then the $P_{\nabla}$ 's form a partition (sector partition) of $\mathbb{Z}^{d}$ and

$$
H_{\mathfrak{m}}^{i}\left(S_{A}\right)=\bigoplus_{\nabla} \mathbb{K}\left[P_{\nabla}\right] \otimes_{\mathbb{K}} H^{i}(\operatorname{Conv}(A), \operatorname{Conv}(A) \backslash \nabla ; \mathbb{K})
$$

On the other hand, for $a \in \mathbb{Z}^{d}$ denote

$$
\nabla(a):=\left\{\text { face } \tau \text { of } \mathbb{R}_{\geq 0} A \mid a \in \mathbb{N} A+\mathbb{Z}(A \cap \tau)\right\}
$$

and consider the equivalence relation $a \equiv a^{\prime} \Leftrightarrow \nabla(a)=\nabla\left(a^{\prime}\right)$. Then $P_{\nabla(a)}$ is the equivalence class containing $a$. Notice that $P_{\nabla}$ could be empty and that $\left[a \in P_{\nabla}\right] \Leftrightarrow\left[P_{\nabla}=P_{\nabla(a)}\right]$.

Theorem 6 in [MM06] shows that the partition determined by the equivalence relation $\sim$ in the proof of Theorem 3.3 is finer than the sector partition determined by $\equiv$.

Notice that each $\mathbb{K}\left[P_{\nabla}\right]$ is naturally a left $\mathbb{Z}^{d}$-graded $D_{A}$-module because each $\mathbb{K}[\mathbb{N} A+\mathbb{Z}(A \cap \tau)]$ is as well. If $S_{A}$ is normal, the $D_{A}$-module $\mathbb{K}\left[P_{\nabla}\right]$ is simple. In fact, we have

Theorem 3.7. If $S_{A}$ is normal and $I$ is a monomial ideal in $S_{A}$, then every simple subquotient of $H_{I}^{i}\left(S_{A}\right)$ is of the form $\mathbb{K}\left[P_{\nabla}\right]$ coming from the sector partition.

Proof. By Theorem 3.3 and Remark 3.6(2), we only have to show that $\sim$ and $\equiv$ define the same equivalence relation on $\mathbb{Z}^{d}$. Note that the normality of $S_{A}$ implies that

$$
\begin{aligned}
& E_{\tau}(a)=\{0\} \text { if } a \in \mathbb{N} A+\mathbb{Z}(A \cap \tau) \text { and } \\
& E_{\tau}(a)=\emptyset \text { if } a \notin \mathbb{N} A+\mathbb{Z}(A \cap \tau) .
\end{aligned}
$$

So we have

$$
\begin{aligned}
& a \sim b \\
\Leftrightarrow & E_{\tau}(a)=E_{\tau}(b) \text { for all } \tau \\
\Leftrightarrow & E_{\tau}(a)=\{0\} \text { if and only if } E_{\tau}(b)=\{0\} \\
\Leftrightarrow & a \in \mathbb{N} A+\mathbb{Z}(A \cap \tau) \text { if and only if } b \in \mathbb{N} A+\mathbb{Z}(A \cap \tau) \\
\Leftrightarrow & a \equiv b .
\end{aligned}
$$

Therefore, the simple subquotients of $\mathbb{K}\left[\mathbb{Z}^{d}\right]$ are precisely the $D_{A}$-modules

$$
\mathbb{K}\left[P_{\nabla}\right]=\bigoplus_{b \in[a]} \mathbb{K} t^{b} \cong \frac{\bigoplus_{b \geq a} \mathbb{K} t^{b}}{\bigoplus_{b>a} \mathbb{K} t^{b}}
$$

Example 3.8. Consider $A=\left(\begin{array}{lll}1 & 1 & 1 \\ 0 & 1 & 2\end{array}\right)$. Then $S_{A}=\mathbb{K}\left[t, t s, t s^{2}\right]$ is a 2-dimensional normal toric algebra. Let $I=(t s)$ be the ideal of $S_{A}$ generated by $t s$. We shall describe a composition series of $H_{I}^{1}\left(S_{A}\right)$. By Čech complex, $H_{I}^{1}\left(S_{A}\right)=\mathbb{K}\left[\mathbb{Z}^{d}\right] / S_{A}$. Following the notation in the proof of Theorem 3.3 and Remark 3.6, let

$$
\begin{aligned}
& \nabla_{0}=\left\{0, \sigma_{1}, \sigma_{2}, \mathbb{R}_{\geq 0} A\right\}, \nabla_{1}=\left\{\sigma_{1}, \mathbb{R}_{\geq 0} A\right\}, \nabla_{2}=\left\{\sigma_{2}, \mathbb{R}_{\geq 0} A\right\} \\
& \nabla_{12}=\left\{\sigma_{1}, \sigma_{2}, \mathbb{R}_{\geq 0} A\right\}, \nabla_{A}=\left\{\mathbb{R}_{\geq 0} A\right\}
\end{aligned}
$$


where $\sigma_{1}=\mathbb{R}_{\geq 0}\left(\begin{array}{l}1 \\ 0\end{array}\right)$ and $\sigma_{2}=\mathbb{R}_{\geq 0}\left(\begin{array}{l}1 \\ 2\end{array}\right)$. Then

$$
\begin{aligned}
& P_{\nabla_{0}}=P_{\nabla\left(a_{0}\right)}=\mathbb{N} A, \\
& P_{\nabla_{1}}=P_{\nabla\left(-a_{1}\right)}=\left[\mathbb{N} A+\mathbb{Z}\left(A \cap \sigma_{1}\right)\right] \backslash\left[\mathbb{N} A+\mathbb{Z}\left(A \cap \sigma_{2}\right)\right], \\
& P_{\nabla_{2}}=P_{\nabla\left(-a_{2}\right)}=\left[\mathbb{N} A+\mathbb{Z}\left(A \cap \sigma_{2}\right)\right] \backslash\left[\mathbb{N} A+\mathbb{Z}\left(A \cap \sigma_{1}\right)\right], \\
& P_{\nabla_{12}}=\emptyset, \text { and } \\
& P_{\nabla_{A}}=P_{\nabla\left(-a_{3}\right)}=\mathbb{Z}^{2} \backslash\left[\left(\mathbb{N} A+\mathbb{Z}\left(A \cap \sigma_{1}\right)\right) \cup\left(\mathbb{N} A+\mathbb{Z}\left(A \cap \sigma_{2}\right)\right)\right],
\end{aligned}
$$

where $a_{0}=\left(\begin{array}{l}0 \\ 0\end{array}\right)$ and $a_{i}, i=1,2,3$, is the $i$-th column of $A$. In terms of notation in Theorem 3.3 ,

$$
\begin{aligned}
T_{a_{0}} & =\mathbb{K}\left[P_{\nabla_{0}}\right]=S_{A}, \\
T_{-a_{1}} & =\mathbb{K}\left[P_{\nabla_{0}}\right] \oplus \mathbb{K}\left[P_{\nabla_{1}}\right], \\
T_{-a_{2}} & =\mathbb{K}\left[P_{\nabla_{0}}\right] \oplus \mathbb{K}\left[P_{\nabla_{2}}\right], \\
T_{-a_{3}} & =\mathbb{K}\left[\mathbb{Z}^{2}\right]=\mathbb{K}\left[P_{\nabla_{0}}\right] \oplus \mathbb{K}\left[P_{\nabla_{1}}\right] \oplus \mathbb{K}\left[P_{\nabla_{2}}\right] \oplus \mathbb{K}\left[P_{\nabla_{A}}\right] .
\end{aligned}
$$

So $0 \subset T_{a_{0}} \subset T_{-a_{1}} \subset T_{-a_{1}}+T_{-a_{2}} \subset T_{-a_{3}}=\mathbb{K}\left[\mathbb{Z}^{2}\right]$ is a composition series of $\mathbb{K}\left[\mathbb{Z}^{2}\right]$. Quotienting out $S_{A}$, we obtain a composition series of $H_{I}^{1}\left(S_{A}\right)$ :

$$
0 \subset \mathbb{K}\left[P_{\nabla_{1}}\right] \subset \mathbb{K}\left[P_{\nabla_{1}}\right] \oplus \mathbb{K}\left[P_{\nabla_{2}}\right] \subset \mathbb{K}\left[P_{\nabla_{1}}\right] \oplus \mathbb{K}\left[P_{\nabla_{2}}\right] \oplus \mathbb{K}\left[P_{\nabla_{A}}\right]
$$

Example 3.9. This example is essentially due to Hartshorne Har70. We adopt its combinatorial description which can be found in ILL+07 or [MS05.

Consider $A=\left(\begin{array}{llll}1 & 1 & 1 & 1 \\ 0 & 1 & 0 & 1 \\ 0 & 0 & 1 & 1\end{array}\right)$. Then $S_{A}=\mathbb{K}[r, r s, r t, r s t]$ is a normal toric algebra. Consider the ideal $I=(r, r s)$ of $S_{A}$. Then the socle $\operatorname{Hom}_{S_{A}}\left(S_{A} / \mathfrak{m}, H_{I}^{2}\left(S_{A}\right)\right)$ is infinite dimensional, where $\mathfrak{m}=(r, r s, r t, r s t)$ is the maximal graded ideal of $S_{A}$. However, according to Theorem $3.3 H_{I}^{2}\left(S_{A}\right)$ is of finite length over $D_{A}$.

In fact, $H_{I}^{2}\left(S_{A}\right)$ is $D_{A}$-simple. To see this, using the notation in Remark 3.6 we consider the filter $\nabla=\left\{\sigma_{12}, \mathbb{R}_{\geq 0} A\right\}$, where $\sigma_{12}=\mathbb{R}_{\geq 0}\left(\begin{array}{ll}1 & 1 \\ 0 & 1 \\ 0 & 0\end{array}\right)$ is a facet of $\mathbb{R}_{\geq 0} A$. We claim that

$$
P_{\nabla}=\left[\mathbb{N} A+\mathbb{Z}\left(A \cap \sigma_{12}\right)\right] \backslash\left[\left(\mathbb{N} A+\mathbb{Z} a_{1}\right) \cup\left(\mathbb{N} A+\mathbb{Z} a_{2}\right)\right],
$$

where $a_{1}=\left(\begin{array}{l}1 \\ 0 \\ 0\end{array}\right)$ and $a_{2}=\left(\begin{array}{l}1 \\ 1 \\ 0\end{array}\right)$. Therefore, in view of the Čech complex we have the isomorphism

$$
H_{I}^{2}\left(S_{A}\right) \cong \mathbb{K}\left[P_{\nabla}\right],
$$

which is $D_{A}$-simple by Theorem 3.7

The claim is equivalent to the equality

$$
\begin{aligned}
& {\left[\mathbb{N} A+\mathbb{Z}\left(A \cap \sigma_{12}\right)\right] \cap\left[\bigcup_{\sigma=\sigma_{13}, \sigma_{24}, \sigma_{34}}(\mathbb{N} A+\mathbb{Z}(A \cap \sigma))\right] } \\
= & {\left[\mathbb{N} A+\mathbb{Z}\left(A \cap \sigma_{12}\right)\right] \cap\left[\left(\mathbb{N} A+\mathbb{Z} a_{1}\right) \cup\left(\mathbb{N} A+\mathbb{Z} a_{2}\right)\right], }
\end{aligned}
$$


where $\sigma_{13}=\mathbb{R}_{\geq 0}\left(\begin{array}{ll}1 & 1 \\ 0 & 0 \\ 0 & 1\end{array}\right), \sigma_{24}=\mathbb{R}_{\geq 0}\left(\begin{array}{ll}1 & 1 \\ 1 & 1 \\ 0 & 1\end{array}\right)$, and $\sigma_{34}=\mathbb{R}_{\geq 0}\left(\begin{array}{ll}1 & 1 \\ 0 & 1 \\ 1 & 1\end{array}\right)$. This equality can be verified by the following data:

$$
\begin{aligned}
& \mathbb{N} A+\mathbb{Z} a_{1}=\left\{{ }^{t}(x, y, z) \in \mathbb{Z}^{3} \mid y \geq 0 \text { and } z \geq 0\right\}, \\
& \mathbb{N} A+\mathbb{Z} a_{2}=\left\{{ }^{t}(x, y, z) \in \mathbb{Z}^{3} \mid x \geq y \text { and } z \geq 0\right\}, \\
& \mathbb{N} A+\mathbb{Z}\left(A \cap \sigma_{12}\right)=\left\{{ }^{t}(x, y, z) \in \mathbb{Z}^{3} \mid z \geq 0\right\}, \\
& \mathbb{N} A+\mathbb{Z}\left(A \cap \sigma_{13}\right)=\left\{{ }^{t}(x, y, z) \in \mathbb{Z}^{3} \mid y \geq 0\right\}, \\
& \mathbb{N} A+\mathbb{Z}\left(A \cap \sigma_{24}\right)=\left\{{ }^{t}(x, y, z) \in \mathbb{Z}^{3} \mid x \geq y\right\}, \\
& \mathbb{N} A+\mathbb{Z}\left(A \cap \sigma_{34}\right)=\left\{{ }^{t}(x, y, z) \in \mathbb{Z}^{3} \mid x \geq z\right\} .
\end{aligned}
$$

Remark 3.10. Helm and Miller HM03 studied the Bass numbers of local cohomology modules over toric algebras. As a generalization of Hartshorne's example, their main result ([HM03], Theorem 7.1) implies that for a Gorenstein normal toric algebra $S_{A}, \mathbb{N} A$ is not simplicial if and only if there exists an $\mathbb{N} A$-graded prime $\mathfrak{p}$ of dimension 2 such that $H_{\mathfrak{p}}^{d-1}\left(S_{A}\right)$ has infinite-dimensional scole.

\section{Associated GRAded Rings gr $D_{A}$ AND CHARACTERIStiC CYCLES}

4.1. Associated graded rings gr $D_{A}$. Let $R$ be a $\mathbb{K}$-algebra as in subsection 2.3 . The definition of $D(R ; \mathbb{K})$ gives an order filtration of $D(R ; \mathbb{K})$ :

$$
0 \subseteq R=D_{0}(R ; \mathbb{K}) \subseteq D_{1}(R ; \mathbb{K}) \subseteq D_{2}(R ; \mathbb{K}) \subseteq \cdots
$$

Define the associated graded ring of $D(R ; \mathbb{K})$ to be

$$
\operatorname{gr} D(R ; \mathbb{K}):=D_{0} \oplus\left(D_{1} / D_{0}\right) \oplus\left(D_{2} / D_{1}\right) \oplus \cdots,
$$

where $D_{i}:=D_{i}(R ; \mathbb{K})$. From the definition of $D(R ; \mathbb{K})$, gr $D(R ; \mathbb{K})$ is a commutative $R$-algebra and we have the natural embedding $R \hookrightarrow \operatorname{gr} D(R ; \mathbb{K})$. For example, if $R=\mathbb{K}\left[t_{1}, \ldots, t_{d}\right]$ is a polynomial algebra over $\mathbb{K}$, then $D(R ; \mathbb{K})=$ $\mathbb{K}\left[t_{1}, \ldots, t_{d}\right]\left\langle\partial_{1}, \ldots, \partial_{d}\right\rangle$ is the Weyl algebra. The associated graded ring gr $D(R ; \mathbb{K})$ $=\mathbb{K}\left[t_{1}, \ldots, t_{d}, \xi_{1}, \ldots, \xi_{d}\right]$ is a $2 d$-dimensional polynomial algebra over $\mathbb{K}$, where $\xi_{i}$ is the image of $\partial_{i}$ in the associated graded $\operatorname{ring} \operatorname{gr} D(R ; \mathbb{K})$. In what follows, we will use the description

$$
\operatorname{gr} D\left(\mathbb{K}\left[t_{1}^{ \pm 1}, \ldots, t_{d}^{ \pm 1}\right] ; \mathbb{K}\right)=\mathbb{K}\left[t_{1}^{ \pm 1}, \ldots, t_{d}^{ \pm 1}, \Theta_{1}, \ldots, \Theta_{d}\right],
$$

where $\Theta_{i}=t_{i} \xi_{i}$ is the image of $\theta_{i}=t_{i} \partial_{i}$ in the associated graded $\operatorname{ring} \operatorname{gr} D(R ; \mathbb{K})$.

When $R$ is a regular algebra over $\mathbb{K}, \operatorname{Spec}(\operatorname{gr} D(R ; \mathbb{K}))$ can be identified as the cotangent bundle of the variety $\operatorname{Spec} R$ with the projection

$$
\pi: \operatorname{Spec}(\operatorname{gr} D(R ; \mathbb{K})) \rightarrow \operatorname{Spec} R
$$

induced by the embedding $R \hookrightarrow \operatorname{gr} D(R ; \mathbb{K})$. The fiber of $\pi$ over a closed point of Spec $R$ is the cotangent space over that point which is isomorphic to the affine space $\mathbb{K}^{\operatorname{dim} R}$.

In this subsection, we discuss the map $\pi$ for a certain class of toric algebras. We shall see that in some cases the fibers of $\pi$ behave nicely (Theorems 4.5, 4.8). We also give an example (Example 4.6) of a more complicated nature.

To begin with, consider the natural order filtration of $D_{A}$ inherited from that of $D\left(\mathbb{K}\left[\mathbb{Z}^{d}\right]\right)$. With respect to this filtration, one can regard $\operatorname{gr} D_{A}$ as a commutative subalgebra of $\operatorname{gr} D\left(\mathbb{K}\left[\mathbb{Z}^{d}\right]\right)=\mathbb{K}\left[t_{1}^{ \pm 1}, \ldots, t_{d}^{ \pm 1}, \Theta_{1}, \ldots, \Theta_{d}\right]$. When $\operatorname{gr} D_{A}$ is finitely generated over $\mathbb{K}$, Musson showed that it has dimension $2 d$ [Mus87]. Saito and 
Traves proved that gr $D_{A}$ is finitely generated over $\mathbb{K}$ if and only if the semigroup $\mathbb{N} A$ is scored [ST04]. By definition, a semigroup $\mathbb{N} A$ is scored if

$$
\mathbb{N} A=\bigcap_{\sigma: \text { facet }}\left\{a \in \mathbb{Z}^{d} \mid F_{\sigma}(a) \in F_{\sigma}(\mathbb{N} A)\right\}
$$

or, equivalently, $\widetilde{\mathbb{N} A} \backslash \mathbb{N} A$ is a union of finitely many hyperplane sections parallel to some facets of $\mathbb{R}_{\geq 0} A$. The scored condition implies Serre's condition $\left(S_{2}\right)$ (see Remark 3.2).

We should remark that if $S_{A}$ is normal, then gr $D_{A}$ is Gorenstein (Mus87, Theorem D). In general, even for a 1-dimensional semigroup ring (which is always scored), the associated graded ring can have bad singularities. In fact, we have the following.

Proposition 4.1. If $S_{A}$ is a 1-dimensional toric algebra which is not normal, then gr $D_{A}$ is not Cohen-Macaulay.

Proof. Using the formula in Lemma 4.3, we see that $\operatorname{gr} D_{A}$ is again a toric algebra over $\mathbb{K}$. Indeed, notice that 0 is the only facet and that $n_{0, w}=|\Omega(w)|$. Note also that $|\Omega(-w)|=w+|\Omega(w)|$ by Lemma 4.4. So by Lemma 4.3.

$$
\operatorname{gr} D_{A}=\mathbb{K}\left[t \xi, t^{|\Omega(w)|} \xi^{|\Omega(-w)|}:|w| \in\left\{a_{1}, \ldots, a_{n}\right\} \cup \operatorname{Hole}(\mathbb{N} A)\right] .
$$

Therefore gr $D_{A}$ is a two-dimensional toric algebra over $\mathbb{K}$.

We claim that

$$
\operatorname{dim}_{\mathbb{K}} \frac{\mathbb{K}[t, \xi]}{\operatorname{gr} D_{A}}<\infty .
$$

Take $\ell$ to be the maximal number of $2|\Omega(-w)|$ for $w \in \operatorname{Hole}(\mathbb{N} A)$. To prove (4.1), it is enough to show that $t^{u} \xi^{v} \in \operatorname{gr} D_{A}$ for all pairs $u, v \in \mathbb{N}$ satisfying $u+v \geq \ell$. Since $\left[t^{u} \xi^{v} \in \operatorname{gr} D_{A} \Leftrightarrow t^{v} \xi^{u} \in \operatorname{gr} D_{A}\right]$, by symmetry we may assume $w_{0}:=u-v \geq 0$.

- If $w_{0} \in \operatorname{Hole}(\mathbb{N} A), 2 u \geq u+v \geq \ell \geq 2\left|\Omega\left(-w_{0}\right)\right|$. So $(t \xi)^{u-\left|\Omega\left(-w_{0}\right)\right|} \in \operatorname{gr} D_{A}$, and hence $t^{u} \xi^{v}=(t \xi)^{u-\left|\Omega\left(-w_{0}\right)\right|} t^{\left|\Omega\left(-w_{0}\right)\right|} \xi^{\left|\Omega\left(w_{0}\right)\right|} \in \operatorname{gr} D_{A}$.

- If $w_{0} \in \mathbb{N} A$ we have $t^{u} \xi^{v}=(t \xi)^{v} t^{w_{0}}=(t \xi)^{v} t^{\left|\Omega\left(-w_{0}\right)\right|} \in \operatorname{gr} D_{A}$.

So the claim is proved. Now, applying the criterion in Remark 3.2 to the toric algebra $\operatorname{gr} D_{A}$, we see that $\operatorname{gr} D_{A}$ doesn't satisfy Serre's condition $\left(S_{2}\right)$. Hence, gr $D_{A}$ is not Cohen-Macaulay.

Remark 4.2. The claim (4.1) holds true for any affine curve with injective normalization (see the proof of Theorem 3.12 in SS88). In fact, this codimension is known to be the Letzter-Makar-Limanov invariant, which plays an important role in the theory of Calogero-Moser space. For more information, see the work by Berest and Wilson BW99.

Now, let $\mathfrak{m}$ be the maximal graded ideal of $S_{A}$ corresponding to the closed point 0 of the toric variety $\operatorname{Spec}\left(S_{A}\right)$. Let $I=\sqrt{\mathfrak{m g r} D_{A}}$ be the radical of the extended ideal of $\mathfrak{m}$ under the embedding $S_{A} \hookrightarrow \operatorname{gr} D_{A}$. We are going to show that if $\mathbb{N} A$ is simplicial and scored, then $\operatorname{gr} D_{A} / I$ is isomorphic to $S_{A}$ as $\mathbb{K}$-algebras. This implies that the reduced induced structure of the fiber of $\pi: \operatorname{Spec}\left(\operatorname{gr} D_{A}\right) \rightarrow \operatorname{Spec}\left(S_{A}\right)$ over the point 0 is isomorphic to the ambient toric variety. 
The following two lemmas are needed:

Lemma 4.3 ([ST04). For scored $\mathbb{N} A$,

$$
\begin{gathered}
\operatorname{gr} D_{A}=\bigoplus_{a \in \mathbb{Z}^{d}} t^{a} \mathbb{K}\left[\Theta_{1}, \ldots, \Theta_{d}\right] \cdot P_{a} \text { where } \\
P_{a}=\prod_{\sigma \in \mathscr{F}} F_{\sigma}(\Theta)^{n_{\sigma, a}} \text { and } n_{\sigma, a}=\#\left\{F_{\sigma}(\mathbb{N} A) \backslash\left[-F_{\sigma}(a)+F_{\sigma}(\mathbb{N} A)\right]\right\} .
\end{gathered}
$$

Lemma 4.4. Let $\mathbb{N} A$ be scored. Then for any $a \in \mathbb{Z}^{d}$ and $\sigma \in \mathscr{F}$,

$$
n_{\sigma,-a}=n_{\sigma, a}+F_{\sigma}(a) .
$$

In particular,

(1) if $\sigma$ is a facet with the property that $F_{\sigma}(a) \leq 0$, then $n_{\sigma, k a} \leq k \cdot n_{\sigma, a}$ for large $k \in \mathbb{N}$, and furthermore $P_{a}^{k}=P_{k a} \cdot P$ for some $P \in \mathbb{K}[\Theta]$;

(2) if $F_{\sigma}(a) \leq 0$ for all $\sigma \in \mathscr{F}$ and $-a \notin \mathbb{N} A$, then $n_{\sigma, k a}<k \cdot n_{\sigma, a}$ for some $\sigma \in \mathscr{F}$ and large $k \in \mathbb{N}$;

(3) if $a \in \mathbb{N} A$, then $n_{\sigma,-a}=F_{\sigma}(a)$;

(4) if $F_{\sigma}(a)>0$, then $n_{\sigma, k a}=0$ for large $k \in \mathbb{N}$.

Proof. To prove $n_{\sigma,-a}=n_{\sigma, a}+F_{\sigma}(a)$ for any $a \in \mathbb{Z}^{d}$ and $\sigma \in \mathscr{F}$, it's enough to show the case where $F_{\sigma}(a)>0$. Set $N=F_{\sigma}(\mathbb{N} A)$ and $n=F_{\sigma}(a)>0$. Then

$$
\begin{aligned}
n_{\sigma,-a} & =\#\{N \backslash(n+N)\} \\
& =\#\{b \in N \mid b-n \notin N\} \\
& =n+\#\{b \in N \mid b-n \notin N \text { but } b-k n \in N \text { for some } k \geq 2\} \\
& =n+\#\{c \in N \mid c+n \notin N\} \\
& =n+\#\{N \backslash(-n+N)\}=n+n_{\sigma, a} .
\end{aligned}
$$

Note that for the second equality we need the assumption that $\mathbb{N} A$ is scored.

Now, we prove the four additional statements:

(1) Notice that $n_{\sigma, k a}=k F_{\sigma}(-a)$ for large $k$ and that $n_{\sigma, a}=n_{\sigma,-a}+F_{\sigma}(-a)$ where $n_{\sigma,-a} \geq 0$.

(2) By assumption, $-a$ lies on a hyperplane parallel to some facet, say $\sigma_{0}$. Then $n_{\sigma_{0},-a}>0$ and hence $n_{\sigma_{0}, k a}<k \cdot n_{\sigma_{0}, a}$ by (1).

(3) $a \in \mathbb{N} A$ implies $n_{\sigma, a}=0$.

(4) This follows from the definition. Indeed, since $\mathbb{N} A$ is scored, $\left(F_{\sigma}(k a)+\mathbb{N}_{0}\right) \subseteq$ $F_{\sigma}(\mathbb{N} A)$ for large $k$. Then $F_{\sigma}(k a)+F_{\sigma}(\mathbb{N} A) \subseteq F_{\sigma}(\mathbb{N} A)$, and hence $n_{\sigma, k a}=0$.

Theorem 4.5. If $\mathbb{N} A$ is a simplicial scored semigroup, then

$$
\operatorname{gr} D_{A} / I=\mathbb{K}\left[\overline{t^{-a_{i}} \cdot P_{-a_{i}}} ; i=1, \ldots, n\right] \cong S_{A},
$$

where $I=\sqrt{\mathfrak{m g r} D_{A}}$ and $\overline{t^{-a_{i}} \cdot P_{-a_{i}}}$ is the image of $t^{-a_{i}} \cdot P_{-a_{i}}$ in $\operatorname{gr} D_{A} / I$.

Proof. We sketch how the proof of the left equality goes. Let $\mathscr{F}=\left\{\sigma_{1}, \ldots, \sigma_{d}\right\}$ be the set of all facets of $\mathbb{R}_{\geq 0} A$, and let

$$
C=-\widetilde{\mathbb{N} A}=\left\{a \in \mathbb{Z}^{d} \mid F_{\sigma}(a) \leq 0 \text { for all } \sigma \in \mathscr{F}\right\} .
$$


We will prove the left equality in three steps. The first step shows $\Theta_{i} \in I$ for $i=1, \ldots, d$. The second step shows $t^{a} \cdot P_{a} \in I$ for all $a \in \mathbb{Z}^{d} \backslash C$. Finally, the third step shows that $t^{a} \cdot P_{a} \in I$ if $a \in C \backslash(-\mathbb{N} A)$ and that $t^{a} \cdot P_{a}$ is a product of some $t^{-a_{i}} \cdot P_{-a_{i}}, i=1, \ldots, n$, if $a \in C \cap(-\mathbb{N} A \backslash\{0\})$.

(1) For each $i=1, \ldots, d$, consider the following subset of $\mathbb{Z}^{d}$ :

$$
\left\{F_{\sigma_{i}}(\Theta)=-1\right\} \cap\left[\bigcap_{j \neq i}\left\{F_{\sigma_{j}}(\Theta)=0\right\}\right] \text {. }
$$

Since $\mathbb{N} A$ is simplicial, this is a one point set for each $i$, say $\left\{u_{i}\right\}$. Notice that since $t^{-u_{i}} \in I, F_{\sigma_{i}}^{n_{\sigma_{i}, u_{i}}}=P_{u_{i}}=t^{-u_{i}} \cdot t^{u_{i}} P_{u_{i}} \in I$, where $n_{\sigma_{i}, u_{i}}>0$. Therefore, $F_{\sigma_{i}} \in I$ for each $i$. Since $F_{\sigma_{1}}, \ldots, F_{\sigma_{d}}$ are linearly independent, we conclude that $\Theta_{i} \in I$ for $i=1, \ldots, d$.

(2) For $a \in \mathbb{Z}^{d} \backslash C, F_{\sigma}(a)>0$ for some $\sigma \in \mathscr{F}$. By Lemma 4.4(4), choose $k$ large so that

$$
P_{k a}=\prod_{F_{\sigma}(a)<0} F_{\sigma}^{n_{\sigma, k a}} .
$$

Now, consider as in (1) the one-point set

$$
\left[\bigcap_{F_{\sigma}(a)<0}\left\{F_{\sigma}(\Theta)=F_{\sigma}(k a)\right\}\right] \cap\left[\bigcap_{F_{\sigma}(a) \geq 0}\left\{F_{\sigma}(\Theta)=0\right\}\right]=\{b\} .
$$

We have $P_{b}=P_{k a}$ and $t^{k a-b} \in I$. By Lemma 4.4(1)

$$
\left(t^{a} P_{a}\right)^{k}=t^{k a} P_{a}^{k}=t^{k a} P_{k a} \cdot P=\left(t^{b} P_{b}\right) \cdot P \cdot t^{k a-b} \in I .
$$

Therefore, $t^{a} P_{a} \in I$ as desired.

(3) Let $a \in C$.

If $-a \notin \mathbb{N} A$, by Lemma 4.4 (2) $\left(t^{a} P_{a}\right)^{k}=t^{k a} P_{k a} \cdot P$ for some nonconstant $P \in \mathbb{K}[\Theta]$. Since $P$ is a product of some $F_{\sigma}$ 's, $P \in I$ by (1), and hence $t^{a} P_{a} \in I$.

If $-a \in \mathbb{N} A \backslash\{0\}$, write $-a=\sum m_{i} a_{i}$. By Lemma 4.4(3),

$$
n_{\sigma, a}=F_{\sigma}(-a)=\sum m_{i} F_{\sigma}\left(a_{i}\right)=\sum m_{i} n_{\sigma,-a_{i}}
$$

and therefore

$$
t^{a} P_{a}=t^{\sum m_{i}\left(-a_{i}\right)} \cdot \prod_{\sigma \in \mathscr{F}} F_{\sigma}^{\sum m_{i} n_{\sigma,-a_{i}}}=\prod\left(t^{-a_{i}} P_{-a_{i}}\right)^{m_{i}}
$$

To complete the proof, we establish the right isomorphism. First, recall that if $R \rightarrow S$ is a homomorphism of commutative rings and $Q$ is a prime ideal in $S$ lying over a prime ideal $q$ of $R$, then $\operatorname{dim}\left(S_{Q} / q S_{Q}\right) \geq \operatorname{ht} Q-\mathrm{ht} q$. On the other hand, since gr $D_{A}$ is finitely generated as a $\mathbb{K}$-algebra which is also a domain, each maximal ideal of gr $D_{A}$ has height $2 d$. (Here, we use the fact that $\operatorname{dim} \operatorname{gr} D_{A}=2 d$.) Therefore, $\operatorname{dim}\left(\operatorname{gr} D_{A} / I\right) \geq d$. Now, consider the surjection from the polynomial ring $\mathbb{K}\left[x_{1}, \ldots, x_{n}\right]$ to $\mathbb{K}\left[\overline{t^{-a_{i}} \cdot P_{-a_{i}}} ; i=1, \ldots, n\right]$. By Lemma 4.4 (3), $P_{-a_{i}}=\prod_{\sigma \in \mathscr{F}} F_{\sigma}^{F_{\sigma}\left(a_{i}\right)}$. Observe that $\overline{t^{-a_{i}} \cdot P_{-a_{i}}}, i=1, \ldots, n$, satisfy the relations in the toric ideal $I_{A}=\left\{x^{u}-x^{v} \mid A u=A v\right\}$ (where for $u \in \mathbb{Z}^{n}, x^{u}:=x_{1}^{u_{1}} \cdots x_{n}^{u_{n}}$ ). 
Hence we have a surjection

$$
S_{A} \cong \mathbb{K}\left[x_{1}, \ldots, x_{n}\right] / I_{A} \longrightarrow \mathbb{K}\left[\overline{t^{-a_{i}} \cdot P_{-a_{i}}} ; i=1, \ldots, n\right],
$$

which is an isomorphism by comparing the dimensions.

Example 4.6. Consider

$$
A=\left(\begin{array}{cccc}
1 & 0 & 0 & 1 \\
0 & 1 & 0 & 1 \\
0 & 0 & 1 & -1
\end{array}\right) .
$$

$S_{A}=\mathbb{K}\left[s, t, u, s t u^{-1}\right]$ is a 3 -dimensional normal toric algebra which is isomorphic to the toric algebra appearing in Example 3.9. By 4.1, 4.6, and 6.3 in [ST04,

$$
\begin{gathered}
\operatorname{gr} D_{A}=\mathbb{K}\left[s, t, u, s t u^{-1}, \Theta_{s}, \Theta_{t}, \Theta_{u}, s^{-1} \Theta_{s}\left(\Theta_{s}+\Theta_{u}\right), t^{-1} \Theta_{t}\left(\Theta_{t}+\Theta_{u}\right), s^{-1} t^{-1} u \Theta_{s} \Theta_{t}\right. \\
\left.u^{-1}\left(\Theta_{s}+\Theta_{u}\right)\left(\Theta_{t}+\Theta_{u}\right), t u^{-1}\left(\Theta_{s}+\Theta_{u}\right), t^{-1} u \Theta_{t}, s u^{-1}\left(\Theta_{t}+\Theta_{u}\right), s^{-1} u \Theta_{s}\right] .
\end{gathered}
$$

Set

$$
\begin{aligned}
& a=s, b=t, c=u, d=s t u^{-1}, e=\Theta_{s}, f=\Theta_{t}, g=\Theta_{u}, h=s^{-1} \Theta_{s}\left(\Theta_{s}+\Theta_{u}\right), \\
& i=t^{-1} \Theta_{t}\left(\Theta_{t}+\Theta_{u}\right), j=u^{-1}\left(\Theta_{s}+\Theta_{u}\right)\left(\Theta_{t}+\Theta_{u}\right), k=s^{-1} t^{-1} u \Theta_{s} \cdot \Theta_{t}, \\
& l=t u^{-1}\left(\Theta_{s}+\Theta_{u}\right), m=t^{-1} u \Theta_{t}, n=s u^{-1}\left(\Theta_{t}+\Theta_{u}\right), o=s^{-1} u \Theta_{s} .
\end{aligned}
$$

Consider the surjection $\phi: \mathbb{K}[a, \ldots, o] \rightarrow \operatorname{gr} D_{A}$. Using Macaulay 2, we see that a primary decomposition of $\sqrt{\mathfrak{m g r} D_{A}}$ is the intersection of the two ideals $\left(o, n, d, a, c, f+g, e, b, f j-i l, f i+j m, f^{2}+l m, f k+h m, h i-j k, f h-k l\right)$ and $\left(m, l, d, a, c, f, e+g, b, g k-i o, g i+k n, g^{2}+n o, g j+h n, h i-j k, g h-j o\right)$ modulo Ker $\phi$. Therefore, the fiber $\pi^{-1}(0)$ has two components, each of which is 4-dimensional.

Remark 4.7. The left equality of Theorem 4.5 and Example 4.6 can be achieved alternatively by a result of Saito.

By Proposition 4.14 in Sai10, $\pi^{-1}(\mathfrak{m})=\left\{\mathfrak{P}(\mathfrak{q}, \nu) \mid \nu \cap \mathbb{R}_{\geq 0} A=\{0\}\right\}$. If $\mathbb{N} A$ is simplicial, then $\pi^{-1}(\mathfrak{m})=\left\{\mathfrak{P}\left(\mathfrak{m}_{0},-\mathbb{R}_{\geq 0} A\right)\right\}$, which is the left equality of Theorem 4.5. On the other hand, consider Example 4.6. If $\mathfrak{P}(\mathfrak{q}, \nu) \in \pi^{-1}(\mathfrak{m})$, then by Sai10], Proposition 4.14, $\mathfrak{q} \supseteq\left(\Theta_{s}, \Theta_{t}+\Theta_{u}\right)$ or $\left(\Theta_{t}, \Theta_{s}+\Theta_{u}\right)$. If $\mathfrak{q}=\left(\Theta_{s}, \Theta_{t}+\Theta_{u}\right)$, then $\nu=\left\{\Theta_{s} \leq 0, \Theta_{t}+\Theta_{u} \leq 0\right\}$. If $\mathfrak{q}=\left(\Theta_{t}, \Theta_{s}+\Theta_{u}\right)$, then $\nu=\left\{\Theta_{t} \leq 0, \Theta_{s}+\Theta_{u} \leq\right.$ $0\}$. They are the two minimal primes mentioned in Example 4.6.

As a corollary of Theorem 4.5, we can describe the fibers $\pi^{-1}(p)$ for every nonzero closed point $p$ in $\operatorname{Spec} S_{A}$. Let $p$ be in the torus orbit $O_{\tau}$ for some $e$-dimensional face $\tau$ of $\mathbb{R}_{\geq 0} A$, so $p$ corresponds to a semigroup homomorphism $f_{p}: \mathbb{N} A \rightarrow \mathbb{K}$ with $f_{p}\left(a_{i}\right)=c_{i}$, where $c_{i}=0$ if and only if $a_{i} \notin \mathbb{N}(A \cap \tau)$. Then $p$ corresponds to the maximal ideal $\mathfrak{m}_{p}=\left(t^{a_{1}}-c_{1}, \ldots, t^{a_{n}}-c_{n}\right)$ of $S_{A}$. The following theorem gives the reduced induced structure of $\pi^{-1}(p)$.

Theorem 4.8. Under the hypotheses of Theorem 4.5, we have

$$
\frac{\operatorname{gr} D_{A}}{\sqrt{\mathfrak{m}_{p} \operatorname{gr} D_{A}}} \cong S_{B} \otimes \mathbb{K}\left[\delta_{1}, \ldots, \delta_{e}\right]
$$

where $S_{B}$ is the toric algebra generated by a simplicial scored semigroup

$$
\mathbb{N} B \cong \frac{\mathbb{N} A+\mathbb{Z}(A \cap \tau)}{\mathbb{Z}(A \cap \tau)}
$$

and $\mathbb{K}\left[\delta_{1}, \ldots, \delta_{e}\right]$ is a polynomial ring in $e(=\operatorname{dim} \tau)$ variables. 
Proof. First, notice that $t^{a}, a \in \mathbb{N}(A \cap \tau)$, acts as a unit on $\frac{\operatorname{gr} D_{A}}{\sqrt{\mathfrak{m}_{p} \operatorname{gr} D_{A}}}$ because $p \in O_{\tau}$. So, by abusing the notation

$$
\frac{\operatorname{gr} D_{A}}{\sqrt{\mathfrak{m}_{p} \operatorname{gr} D_{A}}} \cong \frac{\operatorname{gr} D_{A}\left[\tau^{-1}\right]}{\sqrt{\mathfrak{m}_{p} \operatorname{gr} D_{A}\left[\tau^{-1}\right]}}
$$

where $\tau^{-1}$ means we invert $t^{a}$ for all $a \in \mathbb{N}(A \cap \tau)$. Note also that

$$
\operatorname{gr} D_{A}\left[\tau^{-1}\right] \cong \operatorname{gr} D\left(S_{A}\left[\tau^{-1}\right]\right)=\operatorname{gr} D(\mathbb{K}[\mathbb{N} A+\mathbb{Z}(A \cap \tau)]) .
$$

Next, choose a simplicial scored semigroup $\mathbb{N} B$ so that

$$
\mathbb{N} A+\mathbb{Z}(A \cap \tau)=\mathbb{N} B \oplus \mathbb{Z}(A \cap \tau) .
$$

To do this, let's first assume $\mathbb{N} A$ is normal. By an exercise of section 1.2 in Ful93, $\frac{\mathbb{R} \geq 0}{\mathbb{R} \tau}$ is a simplicial rational polyhedral cone with facets $\frac{\gamma+\mathbb{R} \tau}{\mathbb{R} \tau}$, where the $\gamma$ 's are the facets of $\mathbb{R}_{\geq 0} A$ containing $\tau$. So $\frac{\mathbb{N} A+\mathbb{Z}(A \cap \tau)}{\mathbb{Z}(A \cap \tau)}$ is a simplicial normal semigroup in $\mathbb{Z}^{d} / \mathbb{Z}(A \cap \tau)$. $\mathbb{N} B$ can be obtained by choosing suitable elements in $[\mathbb{N} A+\mathbb{Z}(A \cap$ $\tau)] \backslash \mathbb{Z}(A \cap \tau)$. For the general simplicial scored semigroup $\mathbb{N} A$, we just have to notice that $\widetilde{\mathbb{N} A} \backslash \mathbb{N} A$ is a union of hyperplane sections parallel to some facets of $\mathbb{R}_{\geq 0} A$. So $\widetilde{\mathbb{Z}(A \cap \tau)}=\mathbb{Z}(A \cap \tau)$ and $\left.[\mathbb{N} A \widetilde{\mathbb{Z}(A} \cap \tau)\right] \backslash[\mathbb{N} A+\mathbb{Z}(A \cap \tau)]$ is a union of hyperplane sections parallel to some facets of $\mathbb{R}_{\geq 0} A$ containing $\tau$.

Now,

$$
\operatorname{gr} D_{A}\left[\tau^{-1}\right] \cong \operatorname{gr} D(\mathbb{K}[\mathbb{N} B \oplus \mathbb{Z}(A \cap \tau)]) \cong \operatorname{gr} D_{B} \otimes \operatorname{gr} D_{\mathbb{Z}(A \cap \tau)} .
$$

Therefore,

$$
\begin{aligned}
\frac{\operatorname{gr} D_{A}}{\sqrt{\mathfrak{m}_{p} \operatorname{gr} D_{A}}} \cong \frac{\operatorname{gr} D_{B} \otimes \operatorname{gr} D_{\mathbb{Z}(A \cap \tau)}}{\sqrt{\mathfrak{m}_{p} \operatorname{gr} D_{B} \otimes \operatorname{gr} D_{\mathbb{Z}(A \cap \tau)}}} \\
\cong \frac{\operatorname{gr} D_{B}}{\sqrt{\mathfrak{m}_{B} \operatorname{gr} D_{B}}} \otimes \mathbb{K}\left[\delta_{1}, \ldots, \delta_{e}\right] \\
\cong S_{B} \otimes \mathbb{K}\left[\delta_{1}, \ldots, \delta_{e}\right]
\end{aligned}
$$

by Theorem 4.5, where $\delta_{1}, \ldots, \delta_{e}$ are the standard derivations of $\mathbb{K}[\mathbb{Z}(A \cap \tau)]$.

4.2. Characteristic cycles. Let $D:=D(R ; \mathbb{K})$ as defined in section 2. Let $M$ be a $D$-module with a filtration $\left\{M_{i}\right\}$ such that $D_{i} M_{j} \subseteq M_{i+j}$. The associated graded module gr $M:=\bigoplus M_{i} / M_{i-1}$ has the natural gr $D$-module structure. We call $\left\{M_{i}\right\}$ a good filtration if gr $M$ is finitely generated over gr $D$. If $M$ is finitely generated over $D$ by $x_{1}, \ldots, x_{n}$, then the filtration $\left\{\sum_{j=1}^{n} D_{i} x_{j}\right\}$ is good.

From now on, we assume that gr $D$ is Noetherian. This is always the case when $R$ is regular.

For a $D$-module $M$ with a good filtration $\left\{M_{i}\right\}$, define the characteristic variety $\mathrm{Ch}(M)$ of $M$ to be the support of the gr $D$-module gr $M$,

$$
\text { i.e. } \operatorname{Ch}(M)=\operatorname{Var}\left(\operatorname{ann}_{\operatorname{gr} D} \operatorname{gr} M\right) \subseteq \operatorname{Spec}(\operatorname{gr} D) .
$$

The characteristic cycle $\mathrm{CC}(M)$ of $M$ is the formal sum of the irreducible components $V_{i}$ of $\mathrm{Ch}(M)$ counted with multiplicity. More precisely,

$$
\mathrm{CC}(M)=\sum m_{i} V_{i}
$$

where the multiplicity $m_{i}$ is the length of the $(\operatorname{gr} D)_{p_{i}}$-module $(\operatorname{gr} M)_{p_{i}}$ and $p_{i}$ is the prime ideal corresponding to $V_{i}$. 
$\mathrm{Ch}(M)$ and $\mathrm{CC}(M)$ do not depend on the choice of good filtration. A more detailed discussion about characteristic varieties can be found in Gin86.

Example 4.9. $R$ is naturally a $D$-module generated by the identity 1 . With the filtration $\left\{D_{i} \cdot 1\right\}$, gr $R=R$ is the gr $D$-module generated by 1 . So $R \cong$ gr $D / \operatorname{ann}_{\operatorname{gr} D}(1)$, and therefore $\operatorname{Ch}(R)=\operatorname{Var}\left(\operatorname{ann}_{\operatorname{gr} D}(1)\right)$ is abstractly isomorphic to the ambient variety $\operatorname{Spec} R$.

As we mentioned in the introduction, many invariants of $H_{I}^{i}(R)$ can be computed via the characteristic cycles when $R$ is a polynomial algebra over $\mathbb{K}$ (see e.g. [ÁM00], ÁM04). In this subsection, we compute the characteristic cycles of some local cohomology modules $H_{I}^{i}\left(S_{A}\right)$ using our results of finiteness properties in section 3 . By the main result in [ST04, gr $D_{A}$ is finitely generated over $\mathbb{K}$ if and only if $\mathbb{N} A$ is scored. In particular, gr $D_{A}$ is Noetherian when $\mathbb{N} A$ is scored, so it makes sense to talk about characteristic cycles in this case.

Example 4.10. For a 1-dimensional toric algebra $S_{A}=\mathbb{K}\left[t^{a_{i}} \mid i=1, \ldots, n\right]$, $\mathrm{Ch}\left(H_{I}^{1}\left(S_{A}\right)\right)$ is particularly simple. As in Proposition 4.1.

$$
\operatorname{gr} D_{A}=\mathbb{K}\left[t \xi, t^{|\Omega(w)|} \xi^{|\Omega(-w)|}:|w| \in\left\{a_{1}, \ldots, a_{n}\right\} \cup \operatorname{Hole}(\mathbb{N} A)\right] .
$$

For any monomial ideal $I \neq 0$ of $S_{A}, H_{I}^{1}\left(S_{A}\right)=\frac{D_{A} \cdot(1 / t)}{S_{A}}$ by Theorem 3.1 Notice that $t^{a_{i}} \in \sqrt{\operatorname{ann}_{\operatorname{gr} D_{A}} \operatorname{gr}\left(H_{I}^{1}\left(S_{A}\right)\right)}$ and that $\xi^{a_{i}} \notin \sqrt{\operatorname{ann}_{\operatorname{gr} D_{A}} \operatorname{gr}\left(H_{I}^{1}\left(S_{A}\right)\right)}$. So by Theorem 4.5,

$$
\frac{\operatorname{gr} D_{A}}{\sqrt{\operatorname{ann}_{\mathrm{gr} D_{A}} \operatorname{gr}\left(H_{I}^{1}\left(S_{A}\right)\right)}}=\mathbb{K}\left[\delta_{i} \mid i=1, \ldots, n\right] \cong S_{A},
$$

where $\delta_{i}$ is the image of $\xi^{a_{i}}$ in $\frac{\operatorname{gr} D_{A}}{\sqrt{\operatorname{ann}_{\operatorname{gr} D_{A}} \operatorname{gr}\left(H_{I}^{1}\left(S_{A}\right)\right)}}$. Therefore, $\operatorname{Ch}\left(H_{I}^{1}\left(S_{A}\right)\right)$ is abstractly isomorphic to the ambient toric variety $\operatorname{Spec}\left(S_{A}\right)$. Furthermore, in view of the exact sequence

$$
0 \rightarrow S_{A} \rightarrow D_{A} \cdot(1 / t) \rightarrow H_{I}^{1}\left(S_{A}\right) \rightarrow 0
$$

we have

$$
\mathrm{CC}\left(S_{A}[1 / f]\right)=\operatorname{Ch}\left(S_{A}\right)+\operatorname{Ch}\left(H_{I}^{1}\left(S_{A}\right)\right) \text { for any monomial } f \in S_{A}
$$

by the additivity of the characteristic cycles on exact sequences. In particular, $\mathrm{Ch}\left(S_{A}[1 / f]\right)$ has two components, each of which is abstractly isomorphic to $\operatorname{Spec}\left(S_{A}\right)$.

Example 4.11. Consider the toric algebra in Example 3.8. Let $p=\Theta_{t}$ and $q=\Theta_{s}$. Then we have

$$
\operatorname{gr} D_{A}=\mathbb{K}\left[t, t s^{2}, t s, t^{-1}(2 p-q)^{2}, s(2 p-q), t^{-1} s^{-1}(2 p-q) q, t^{-1} s^{-2} q^{2}, s^{-1} q, p, q\right] \text {. }
$$

Set

$$
\begin{aligned}
& a=t, b=t s^{2}, c=t s, d=t^{-1}(2 p-q)^{2}, e=s(2 p-q), \\
& f=t^{-1} s^{-1}(2 p-q) q, g=t^{-1} s^{-2} q^{2}, h=s^{-1} q, i=p, j=q,
\end{aligned}
$$

and consider the surjection $\phi: \mathbb{K}[a, \ldots, j] \rightarrow \operatorname{gr} D_{A}$. Table 1 gives the information about the characteristic cycles. Again, notice that each $M$ is cyclic by Theorem 3.1 . so $\operatorname{ann}_{\mathrm{gr}} D_{A} \operatorname{gr} M$ is easy to compute. 
TABLE 1

\begin{tabular}{|c|c|c|}
\hline$M$ & $J=\phi^{-1}\left(\operatorname{ann}_{\operatorname{gr} D_{A}} \operatorname{gr} M\right)$ & primary decomposition of $\sqrt{J}$ \\
\hline$S_{A}[1 / t]$ & $(f, g, h, i, j)+\operatorname{Ker} \phi$ & $\left(a, c, f, g, h, i, f, b d-e^{2}\right) \cap\left(d, e, f, g, h, i, j, a b-c^{2}\right)$ \\
\hline$H_{(t)}^{1}\left(S_{A}\right)$ & $(f, g, h, i, j, a)+\operatorname{Ker} \phi$ & $\left(a, c, f, g, h, i, f, b d-e^{2}\right)$ \\
\hline$S_{A}\left[1 / t s^{2}\right]$ & $(d, e, f, i, j)+\operatorname{Ker} \phi$ & $\left(j, i, h, g, f, e, d, a b-c^{2}\right) \cap\left(j, i, f, c, b, e, d, a g-h^{2}\right)$ \\
\hline$H_{\left(t s^{2}\right)}^{1}\left(S_{A}\right)$ & $(d, e, f, i, j, b)+\operatorname{Ker} \phi$ & $\left(j, i, f, e, d, c, b, a g-h^{2}\right)$ \\
\hline$S_{A}[1 / t s]$ & $(i, j)+\operatorname{Ker} \phi$ & $\begin{array}{c}\left(j, i, h, c, a, b, e, d g-f^{2}\right) \cap\left(j, i, h, g, f, c, a, b d-e^{2}\right) \\
\cap\left(j, i, h, g, f, e, d, a b-c^{2}\right) \cap\left(j, i, f, c, b, e, d, a g-h^{2}\right)\end{array}$ \\
\hline$H_{\left(t, t s^{2}\right)}^{2}\left(S_{A}\right)$ & $(a, b, c, e, h, i, j)+\operatorname{Ker} \phi$ & $\left(a, b, c, e, h, i, j, d g-f^{2}\right)$ \\
\hline
\end{tabular}

Example 4.12. Consider the toric algebra in Example 4.6 and use the notation there. By Theorem 3.1, we see that

$$
J:=\phi^{-1}\left(\operatorname{ann}_{\operatorname{gr} D_{A}} \operatorname{gr}\left(H_{(s)}^{1}\left(S_{A}\right)\right)\right)=(a, e, f, g, i, j, k, m, n)+\operatorname{Ker} \phi
$$

and the primary decomposition of $\sqrt{J}$ is

$$
\begin{aligned}
\sqrt{J}= & (o, n, m, k, j, i, c, a, f, g, h, e) \cap(n, m, l, k, j, i, d, a, f, g, h, e) \\
& \cap(n, m, k, j, i, d, c, a, f, g, e, b h-l o) .
\end{aligned}
$$

So $\mathrm{CC}\left(H_{(s)}^{1}\left(S_{A}\right)\right)$ is a sum of varieties which are not all isomorphic.

To compute $\mathrm{Ch}\left(H_{\mathfrak{m}}^{3}\left(S_{A}\right)\right)$, we need

$$
J^{\prime}:=\phi^{-1}\left(\operatorname{ann}_{\operatorname{gr} D_{A}} \operatorname{gr}\left(H_{\mathfrak{m}}^{3}\left(S_{A}\right)\right)\right)=(a, b, c, d, e, f, g, l, m, n, o)+\operatorname{Ker} \phi
$$

and $\sqrt{J^{\prime}}=(a, b, c, d, e, f, g, l, m, n, o, h i-j k)$.

Inspired by Examples 4.10, 4.11, and 4.12, we have the following.

Theorem 4.13. For any scored pointed semigroup $\mathbb{N} A$, the characteristic variety $\mathrm{Ch}\left(H_{\mathfrak{m}}^{d}\left(S_{A}\right)\right)$ is abstractly isomorphic to the ambient toric variety $\operatorname{Spec}\left(S_{A}\right)$.

Proof. Since $\mathbb{N} A$ is pointed, by the Ishida complex

$$
H_{\mathfrak{m}}^{d}\left(S_{A}\right)=\frac{\mathbb{K}\left[\mathbb{Z}^{d}\right]}{\mathbb{K}\left[\bigcup_{\sigma: f a c e t}(\mathbb{N} A+\mathbb{Z}(A \cap \sigma))\right]} .
$$

By Theorem [3.1, $\mathbb{K}\left[\mathbb{Z}^{d}\right]=D_{A} \cdot\left(1 / t^{\alpha}\right)$ for some interior point $\alpha$, i.e. for some $\alpha \in \mathbb{N} A \backslash\left[\bigcup_{\sigma: \text { facet }} \mathbb{N}(A \cap \sigma)\right]$. Consider the expression of gr $D_{A}$ in Lemma 4.3 . Denote $J:=\operatorname{ann}_{\operatorname{gr} D_{A}} \operatorname{gr}\left(H_{\mathfrak{m}}^{d}\left(S_{A}\right)\right)$. Notice that:

(1) For any $a \in \mathbb{Z}^{d} \backslash(-\widetilde{\mathbb{N} A})$ and for any facet $\sigma$ with $F_{\sigma}(a)>0$, there exists $n \in \mathbb{N}$ so that $n a-\alpha \in \mathbb{N} A+\mathbb{Z}(A \cap \sigma)$. Therefore,

$$
t^{a} \mathbb{K}\left[\Theta_{1}, \ldots, \Theta_{d}\right] \cdot P_{a} \subseteq \sqrt{J}
$$

for all $a \in \mathbb{Z}^{d} \backslash(-\widetilde{\mathbb{N} A})$.

(2) For $a=0 \in \mathbb{Z}^{d}, P_{a}=1$ and $\theta_{i} \cdot t^{-\alpha}=-\alpha_{i} t^{-\alpha} \in H_{\mathfrak{m}}^{d}\left(S_{A}\right)$. So by considering the order filtration, $\Theta_{i} \in J$ for all $i=1, \ldots, d$.

(3) By exactly the same argument as in item (3) in the proof of Theorem 4.5. we have $t^{a} P_{a} \in \sqrt{J}$ for $a \in[-\widetilde{\mathbb{N} A} \backslash(-\mathbb{N} A)]$. Also, for $a \in-\mathbb{N} A \backslash\{0\}, t^{a} P_{a}$ is a product of some $t^{-a_{i}} P_{-a_{i}}$. 
(4) For $a \in-\mathbb{N} A \backslash\{0\}, t^{a} P_{a} \cdot t^{-\alpha}=P(-\alpha) t^{a-\alpha} \neq 0$ in $\operatorname{gr}\left(H_{\mathfrak{m}}^{d}\left(S_{A}\right)\right)$ because $\alpha$ is an interior point and because $a-\alpha \in \mathbb{Z}^{d} \backslash \bigcup_{\sigma: \text { facet }} \mathbb{K}[\mathbb{N} A+\mathbb{Z}(A \cap \sigma)]$. So $t^{a} P_{a} \notin \sqrt{J}$ for $a \in-\mathbb{N} A \backslash\{0\}$.

Therefore,

$$
\frac{\operatorname{gr} D_{A}}{\sqrt{J}}=\mathbb{K}\left[\overline{t^{-a_{i}} P_{-a_{i}}} \mid a_{i}: \text { columns of } A\right],
$$

which is isomorphic to $S_{A}$ by a similar argument as in the final part of the proof of Theorem 4.5.

Remark 4.14. We mention that the description of $\sqrt{J}$ in Theorem 4.13 can be obtained alternatively using Theorem 6.2(2) in Sai10.

For $\beta$, let $\mathfrak{m}_{\beta}$ be the ideal $\left(\theta_{1}-\beta_{1}, \ldots, \theta_{d}-\beta_{d}\right)$ of the polynomial ring $\mathbb{K}\left[\theta_{1}, \ldots, \theta_{d}\right]$. Note that $\mathfrak{m}_{\beta}=\mathfrak{m}_{0}+\beta$ in the notation of [Sai10]. Then $L\left(\mathfrak{m}_{\beta}\right)=D_{A} / I\left(\mathfrak{m}_{\beta}\right)$ is an irreducible $D_{A}$-module (see Theorem 4.1.6 in [ST01] and (5.2) in [Sai10]).

Take $\alpha \in \mathbb{Z}^{d}$ as in the proof of Theorem 4.13. Then we claim that

$$
H_{\mathfrak{m}_{A}}^{d}\left(S_{A}\right) \cong L\left(\mathfrak{m}_{-\alpha}\right) .
$$

Since $\tau\left(\mathfrak{m}_{0}-\alpha\right)=-\mathbb{R}_{\geq 0} A, \sqrt{J}=\sqrt{\operatorname{gr} I\left(\mathfrak{m}_{-\alpha}\right)}=\mathfrak{P}\left(\mathfrak{m}_{0},-\mathbb{R}_{\geq 0} A\right)$ by (4.3) and Theorem 6.2(2) in Sai10.

The claim (4.3) can be proved as follows: By Theorem 3.1 $H_{\mathfrak{m}_{A}}^{d}\left(S_{A}\right)=D_{A} \cdot t^{-\alpha}$. Since $\mathbb{N} A$ is scored, $\mathbb{Z}^{d} \cap \mathbb{Q}(A \cap \tau)=\mathbb{Z}(A \cap \tau)$ for all faces $\tau$. Hence, for $\beta \in \mathbb{Z}^{d}, \beta \nsim$ $-\alpha$ if and only if $\beta \in \mathbb{N} A+\mathbb{Z}(A \cap \tau)$ for some face $\tau$ if and only if $\beta \in \mathbb{N} A+\mathbb{Z}(A \cap \sigma)$ for some facet $\sigma$. Therefore, we see that $I\left(\mathfrak{m}_{-\alpha}\right) \cdot t^{-\alpha}=0$ by (4.2). Thus there exists a surjective $D_{A}$-homomorphism from $L\left(\mathfrak{m}_{-\alpha}\right)$ to $H_{\mathfrak{m}_{A}}^{d}\left(S_{A}\right)$. Since $L\left(\mathfrak{m}_{-\alpha}\right)$ is irreducible, it is an isomorphism.

\section{ACKNOWLEDGMENT}

This work originates from a conversation between Uli Walther and William Traves. The author is grateful to his advisor, Uli Walther, for introducing this problem and providing many useful discussions and comments. He would also like to thank Ezra Miller for the comments on sector partition and Example 3.9, and Christine Berkesch for carefully reading this paper. Special thanks go to the referee of this paper who helped improve the presentation, simplified the proof of Theorem [3.1] and pointed out the relations between our results and the results in Sai10.

\section{REFERENCES}

[ÁM00] Josep Álvarez Montaner, Characteristic cycles of local cohomology modules of monomial ideals, J. Pure Appl. Algebra 150 (2000), no. 1, 1-25. MR1762917(2001d:13016)

[ÁM04] Characteristic cycles of local cohomology modules of monomial ideals. II, J. Pure Appl. Algebra 192 (2004), no. 1-3, 1-20. MR2067186

[ÁMBL05] Josep Álvarez-Montaner, Manuel Blickle, and Gennady Lyubeznik, Generators of D-modules in positive characteristic, Math. Res. Lett. 12 (2005), no. 4, 459-473. MR2155224(2006m:13024)

[Bjö79] J.-E. Björk, Rings of differential operators, North-Holland Mathematical Library, vol. 21, North-Holland Publishing Co., Amsterdam, 1979. MR549189 (82g:32013)

[Bøg95] Rikard Bøgvad, Some results on D-modules on Borel varieties in characteristic $p>0$, J. Algebra 173 (1995), no. 3, 638-667. MR1327873 (97a:14015) 
[Bøg02] _ An analogue of holonomic D-modules on smooth varieties in positive characteristics, Homology Homotopy Appl. 4 (2002), no. 2, part 1, 83-116, The Roos Festschrift volume, 1. MR.1918185(2003h:14030)

[BS98] M. P. Brodmann and R. Y. Sharp, Local cohomology: An algebraic introduction with geometric applications, Cambridge Studies in Advanced Mathematics, vol. 60, Cambridge University Press, Cambridge, 1998. MR1613627 (99h:13020)

[BW99] Yuri Berest and George Wilson, Classification of rings of differential operators on affine curves, Internat. Math. Res. Notices (1999), no. 2, 105-109. MR1670188 (2000f:14025)

[Ful93] William Fulton, Introduction to toric varieties, Annals of Mathematics Studies, vol. 131, Princeton University Press, Princeton, NJ, 1993, The William H. Roever Lectures in Geometry. MR.1234037 (94g:14028)

[Gin86] V. Ginsburg, Characteristic varieties and vanishing cycles, Invent. Math. 84 (1986), no. 2, 327-402. MR833194 (87j:32030)

[Har70] Robin Hartshorne, Affine duality and cofiniteness, Invent. Math. 9 (1969/1970), 145164. MR0257096 (41:1750)

[HM03] David Helm and Ezra Miller, Bass numbers of semigroup-graded local cohomology, Pacific J. Math. 209 (2003), no. 1, 41-66. MR1973933 (2004c:13028)

[HM05] - Algorithms for graded injective resolutions and local cohomology over semigroup rings, J. Symbolic Comput. 39 (2005), no. 3-4, 373-395. MR2168288 (2007d:13025)

[HS93] Craig L. Huneke and Rodney Y. Sharp, Bass numbers of local cohomology modules, Trans. Amer. Math. Soc. 339 (1993), no. 2, 765-779. MR.1124167 (93m:13008)

[ILL+07] Srikanth B. Iyengar, Graham J. Leuschke, Anton Leykin, Claudia Miller, Ezra Miller, Anurag K. Singh, and Uli Walther, Twenty-four hours of local cohomology, Graduate Studies in Mathematics, vol. 87, American Mathematical Society, Providence, RI, 2007. MR2355715(2009a:13025)

[Ish88] Masa-Nori Ishida, The local cohomology groups of an affine semigroup ring, Algebraic geometry and commutative algebra, vol. I, Kinokuniya, Tokyo, 1988, pp. 141-153.

[Jon94] A. G. Jones, Rings of differential operators on toric varieties, Proc. Edinburgh Math. Soc. (2) 37 (1994), no. 1, 143-160. MR.1258039 (95d:16030)

[Lyu93] Gennady Lyubeznik, Finiteness properties of local cohomology modules (an application of D-modules to commutative algebra), Invent. Math. 113 (1993), no. 1, 41-55. MR.1223223 (94e:13032)

[Lyu97] , F-modules: Applications to local cohomology and D-modules in characteristic $p>0$, J. Reine Angew. Math. 491 (1997), 65-130. MR.1476089 (99c:13005)

[Lyu00] , Finiteness properties of local cohomology modules: A characteristic-free approach, J. Pure Appl. Algebra 151 (2000), no. 1, 43-50. MR1770642 (2001g:13038)

[MM06] Laura Felicia Matusevich and Ezra Miller, Combinatorics of rank jumps in simplicial hypergeometric systems, Proc. Amer. Math. Soc. 134 (2006), no. 5, 1375-1381 (electronic). MR 2199183 (2006j:33016)

[MS05] Ezra Miller and Bernd Sturmfels, Combinatorial commutative algebra, Graduate Texts in Mathematics, vol. 227, Springer-Verlag, New York, 2005. MR2110098(2006d:13001)

[Mus87] Ian M. Musson, Rings of differential operators on invariant rings of tori, Trans. Amer. Math. Soc. 303 (1987), no. 2, 805-827. MR902799 (88m:32019)

[Mus94] - Differential operators on toric varieties, J. Pure Appl. Algebra 95 (1994), no. 3, 303-315. MR.1295963 (95i:16026)

[Sai10] Mutsumi Saito, The spectrum of the graded ring of differential operators of a scored semigroup algebra, Comm. in Algebra 38 (2010), 829-847. MR2598902

[SS88] S. P. Smith and J. T. Stafford, Differential operators on an affine curve, Proc. London Math. Soc. (3) 56 (1988), no. 2, 229-259. MR922654 (89d:14039)

[SS90] Uwe Schäfer and Peter Schenzel, Dualizing complexes of affine semigroup rings, Trans. Amer. Math. Soc. 322 (1990), no. 2, 561-582. MR1076179 (92a:13012)

[SS04] Anurag K. Singh and Irena Swanson, Associated primes of local cohomology modules and of Frobenius powers, Int. Math. Res. Not. (2004), no. 33, 1703-1733. MR2058025 (2005d:13030)

[ST01] Mutsumi Saito and William N. Traves, Differential algebras on semigroup algebras, 207-226. MR1874281(2003d:16034) 
[ST04] - Finite generation of rings of differential operators of semigroup algebras, J. Algebra 278 (2004), no. 1, 76-103. MR2068067 (2005e:16041)

[ST09] Mutsumi Saito and Ken Takahashi, Noetherian properties of rings of differential operators of affine semigroup algebras, Osaka J. Math. 46 (2009), no. 2, 529-556. MR 2549600

[TT08] Shunsuke Takagi and Ryo Takahashi, D-modules over rings with finite Frepresentation type, Math. Res. Lett. 15 (2008), no. 3, 563-581. MR2407232 (2009e:13003)

Department of Mathematics, Purdue University, 150 N. University Street, West LAFAYETTE, Indiana 47907

E-mail address: jhsiao@math.purdue.edu 